\title{
Animal Models in the Study of Exercise-Induced Cardiac Hypertrophy
}

\author{
Y. WANG ${ }^{1}$, U. WISLOFF ${ }^{2}$, O. J. KEMI ${ }^{3}$ \\ ${ }^{1}$ Department of Human Kinesiology, Capital Institute of Physical Education, Beijing, China, \\ ${ }^{2}$ Department of Circulation and Medical Imaging, Norwegian University of Science and \\ Technology, Trondheim, Norway, ${ }^{3}$ Institute of Cardiovascular and Medical Sciences, University \\ of Glasgow, United Kingdom
}

Received November 3, 2009

Accepted January 29, 2010

On-line April 20, 2010

\section{Summary}

Exercise training-induced cardiac hypertrophy occurs following a program of aerobic endurance exercise training and it is considered as a physiologically beneficial adaptation. To investigate the underlying biology of physiological hypertrophy, we rely on robust experimental models of exercise training in laboratory animals that mimic the training response in humans. A number of experimental strategies have been established, such as treadmill and voluntary wheel running and swim training models that all associate with cardiac growth. These approaches have been applied to numerous animal models with various backgrounds. However, important differences exist between these experimental approaches, which may affect the interpretation of the results. Here, we review the various approaches that have been used to experimentally study exercise training-induced cardiac hypertrophy; including the advantages and disadvantages of the various models.

\section{Key words}

Animal models • Exercise $\bullet$ Heart $\bullet$ Hypertrophy

\section{Corresponding author}

Ole J Kemi, University of Glasgow, Institute of Cardiovascular and Medical Sciences, West Medical Building, Glasgow, G12 8QQ, United Kingdom. Fax: +44(0)1413302923. E-mail: ole.kemi@glasgow.ac.uk

\section{Introduction}

Cardiac hypertrophy is a central feature in several scenarios of pathological and physiological remodeling. In cardiovascular disease, pathological remodeling with hypertrophy and/or adjacent dilatation leads to heart dysfunction and failure, and is at least partly caused by altered cardiac gene expression and molecular phenotype (Beisvag et al. 2009). Although the response compensates for reduced contractility, the longterm effect is progressive dysfunction. In contrast, physiological hypertrophy and remodeling induced by pregnancy or endurance exercise training (athletes' heart) is considered beneficial, as it supports the increased $\mathrm{O}_{2}$ demand. An increase in left ventricular (LV) mass is commonly observed after endurance exercise training, but examples of athletes showing LV cavity enlargement with end-diastolic dimensions suggesting pathologically dilated cardiomyopathy also occur (Maron and Pelliccia 2006). These observations question the nature of physiologic versus pathologic hypertrophy. For instance, although pressure and volume overloads are linked to pathological hypertrophy, they also occur during exercise training. Our molecular understanding of exerciseinduced hypertrophy is much due to experimental studies in laboratory animal models, since this cannot be studied in humans. Here, we review the various models available for studying endurance exercise-induced hypertrophy and their advantages and disadvantages. Small rodents such 
as rats and mice are most commonly used for these studies, although other models have also been established (Table 1).

\section{Rat and mice models of exercise training}

Rats and mice present with short gestation periods and many offspring, leading to mass production with relative ease. Furthermore, they reach adult age within $\sim 2$ months, are inexpensive, and though several contractile aspects of the myocardium are different from humans, structure and growth patterns are well characterized and bear similarities to human hearts (Hasenfuss 1998).

The use of genetic engineering for studying genetic and molecular pathways of cardiac remodeling and hypertrophy has re-emphasized mice in the study of exercise training. Also, artificially selecting mouse and rat strains for a multifactorial phenotype, for instance the metabolic syndrome (Wisloff et al. 2005), has also broadened the applicability of these species.

Table 1. Animal models of exercise training-induced cardiac hypertrophy.

\section{Species and exercise training program}

\section{Comments}

\section{Selected}

references

\section{Rat treadmill running}

Adult female, $10-20 \%$ grade, 5times/week, $60 \mathrm{~min} /$ day, 11 weeks, $10-26 \mathrm{~m} / \mathrm{min}$

Adult female, $10 \%$ grade, 5 times/week, $60 \mathrm{~min} /$ day, 20-30 weeks, $31 \mathrm{~m} / \mathrm{min}$

Adult male, $0 \%$ grade, 5times/week, $30 \mathrm{~min} /$ day, 6-12weeks, $0.8 \mathrm{~km} / \mathrm{h}$

Adult male+female, $47 \%$ grade, 5 times/week, $60 \mathrm{~min} /$ day, $4-13$ weeks, intervals $8 \mathrm{~min} 85-90 \%$ of $V \mathrm{O}_{2 \max }$ and 2 min $50-60 \%$ of $V \mathrm{O}_{2 \max }$

\section{Rat voluntary wheel running}

Adult female, free access, 6 weeks

\section{Rat swim training}

Young adult male, water $30-32{ }^{\circ} \mathrm{C}$, 5times/week, $75 \mathrm{~min} /$ day, 8 weeks

Adult male, water $30-32{ }^{\circ} \mathrm{C}$, 5times/week, $60 \mathrm{~min} /$ day, 8 weeks, $+5 \% \mathrm{BW}$ load attached to tail

\section{Mouse treadmill running}

Adult male+female, $47 \%$ grade, 5 times/week, $120 \mathrm{~min} /$ day, 8 weeks, intervals $8 \mathrm{~min} 85-90 \%$ of $V \mathrm{O}_{2 \max }$ and $2 \min 50 \%-60 \%$

\section{Mouse voluntary wheel running}

Adult mice various strains, free access, 2 weeks

Adult male, free access, 2-4 weeks
Graded increase velocity and duration:

$\mathrm{LV} / \mathrm{BW} \uparrow$

Constant protocol:

$\mathrm{LV} / \mathrm{BW} \uparrow$, cardiomyocyte dimensions $\uparrow$

Constant protocol:

$\mathrm{LV} / \mathrm{BW} \uparrow$

High-intensity protocol:

$\mathrm{LV} / \mathrm{BW} \uparrow, \mathrm{RV} / \mathrm{BW} \uparrow, \mathrm{HW} / \mathrm{BW} \uparrow$, cardiomyocyte dimensions $\uparrow$

Voluntary protocol: $\mathrm{HW} / \mathrm{BW} \uparrow, \mathrm{LV} / \mathrm{BW} \uparrow, \mathrm{RV} / \mathrm{BW} \uparrow$

Constant protocol:

$\mathrm{LV} / \mathrm{BW} \uparrow$

Loaded protocol:

$\mathrm{LV} / \mathrm{BW} \uparrow, \mathrm{RV} / \mathrm{BW} \uparrow, \mathrm{HW} / \mathrm{BW} \uparrow$

High-intensity protocol:

$\mathrm{LV} / \mathrm{BW} \uparrow, \mathrm{RV} / \mathrm{BW} \uparrow, \mathrm{HW} / \mathrm{BW} \uparrow$, cardiomyocyte dimensions $\uparrow$

Voluntary protocol: strain-specific responses $\mathrm{HW} / \mathrm{BW}$ $\mathrm{C} 3 \mathrm{H} / \mathrm{HeJ} \mathrm{J}$ least response, DBA/2 J greatest response

Voluntary protocol, exercise and running durations recorded: average 4.3 h/day, $6.8 \mathrm{~km} /$ day at week 4 ; HW/BW $\uparrow$
Diffee and Nagle

Moore et al.

Fenning et al.

(2003)

Wisloff et al.

(2001)

Natali et al. (2001)

Iemitsu et al.

Medeiros et al.

(2004)

Kemi et al. (2001)

Lerman et al.

Allen et al. (2001) 


\section{Mouse swim training}

Adult male, water $30-32^{\circ} \mathrm{C}, 1$ vs 2 times/day, 5 days/week, 60 vs $90 \mathrm{~min} /$ time,

4 vs 6 weeks, +2 vs $4 \%$ BW load attached to tail

\section{Swine treadmill running}

Adult Hampshire farm + Yucatan miniature, 5times/week, 30-60 min/day, 10 weeks, continuous or interval running $70-85 \%$ or $100 \%$ of $\mathrm{HR}_{\max }$

Adult female Yucatan miniature, 5times/week, $75 \mathrm{~min} /$ day, 22 weeks, sprint $15 \mathrm{~min}$

5-8 $\mathrm{mph}+60 \mathrm{~min}$ endurance 4-5 $\mathrm{mph}$

\section{Dog sled pulling}

Adult Alaskan sled (mongrel), team pull sled exercise $20 \mathrm{~km} /$ day, 5 months, 30-40\% of $V \mathrm{O}_{2 \max }$

\section{Rabbit treadmill}

Adult New Zealand white, 5times/week, 40-60 $\mathrm{min} /$ day, 12 weeks, $18-20 \mathrm{~m} / \mathrm{min}$

Effect of different durations,
frequencies and loads: $\mathrm{LV} / \mathrm{BW} \uparrow$,
$\mathrm{HW} / \mathrm{BW} \uparrow$ dependent on protocol

Intensity-dependent effects:

$\mathrm{LV} / \mathrm{BW} \uparrow$

Graded protocol:

$\mathrm{HW} / \mathrm{BW} \uparrow$

Constant loaded protocol:

LV mass index $\uparrow, \mathrm{LV}$ diameter $\uparrow$

Indices of cardiac hypertrophy $\uparrow$

Constant protocol:

HW/BW $\uparrow$, cardiomyocyte

dimensions $\uparrow$, involvement of Aktpathway

High-intensity protocol:

$\mathrm{HW} / \mathrm{BW} \uparrow$, cardiomyocyte

dimensions $\uparrow$, greater response in

normal than metabolic syndrome
Evangelista et al.

White et al. (1997)

Laughlin et al.

Stepien et al.

Carroll and Kyser

McMullen et al.

Wisloff et al. $47 \%$ grade, 5times/week, $60 \mathrm{~min} /$ day, 8 weeks, intervals $8 \mathrm{~min} 85-90 \%$ of $V \mathrm{O}_{2 \max }$ and $2 \mathrm{~min}$ $50-60 \%$ of $V \mathrm{O}_{2 \max }$

HW - heart weight, BW - body weight, LV - left ventricle, RV - right ventricle, TL - tibial length, $\mathrm{V}_{2 \max }$ - maximal oxygen uptake, $\mathrm{HR}_{\max }$ - maximal heart rate, arrow indicates increase.

\section{Treadmill running}

Different treadmill running protocols have been developed, lasting from weeks to months, with individual running session durations ranging from minutes to hours and running speeds ranging $10-97 \mathrm{~m} / \mathrm{min}$, and with the treadmill inclinations ranging 0-25 $(0-47 \%)$ (Fenning et al. 2003, Kemi et al. 2002, Wisloff et al. 2001, Zhang et al. 2002).

Most studies in rats and mice have applied continuous treadmill running, characterized by fixed or progressively increasing speed, inclination, and duration during the session. In rats, these protocols increase heart:body weight ratios up to $30 \%$ (Diffee and Nagle 2003, Fenning et al. 2003, Moore et al. 1993), but have also failed to induce cardiac hypertrophy, despite long exercise periods (Moran et al. 2003). In mice, continuous running protocols have induced only a limited degree of cardiac hypertrophy, either observed as modest increases in ventricular mass or cardiomyocyte dimensions (Bellafiore et al. 2007, Rosa et al. 2005), or no hypertrophy at all (Fewell et al. 1997). The reason for the varied results is unknown. However, the relative exercise load during an exercise training period decreases if the absolute load is kept constant as the exercise capacity (maximal oxygen uptake; $V \mathrm{O}_{2 \max }$ ) increases. This may potentially obscure the response to exercise training. Therefore, the exercise training intensity should be set relative to the individual fitness level.

Interval training models have been used progressively more for studying exercise-induced cardiac hypertrophy and adaptation. This mode of exercise allows for high-intensity running bouts, in which exercise time in the high intensity zone is accumulated over time; the argument being that high aerobic intensity appears more 
effective than lower intensities for inducing structural and functional adaptations to the heart (Haram et al. 2009, Kemi et al. 2005). Interval training by successive 4- to 8min high-intensity treadmill running bouts at $90 \%$ of $V \mathrm{O}_{2 \max }$; achieved by running speeds of $>30 \mathrm{~m} / \mathrm{min}$ on a $25^{\circ}$ inclined treadmill, interspersed by 2 -min lowintensity intervals $\left(\sim 50 \%\right.$ of $\left.V \mathrm{O}_{2 \max }\right)$, induced observable hypertrophy within 4 weeks, and resulted in 25-35\% increased LV and right ventricular (RV) weights, and $\sim 15 \%$ increased cardiomyocyte dimensions after 7-13 weeks of exercise training (Kemi et al. 2002, 2005, 2008, Wisloff et al. 2001, 2002). This is superior to continuous (Iemitsu et al. 2006, Moore et al. 1993) and intensitycontrolled moderate intensity treadmill running programs at $65-70 \%$ of $V \mathrm{O}_{2 \max }$ (Haram et al. 2009, Kemi et al. 2005). The exercise intensity in these studies was controlled by weekly measures of $V \mathrm{O}_{2 \max }$, whereby the running speed was adjusted to maintain constant relative exercise intensity. Only guidance by $V \mathrm{O}_{2 \max }$ can achieve this (Hoydal et al. 2007).

A different approach to interval running, by reducing the duration and increasing the speed of each running bout well into anaerobic intensities $(97 \mathrm{~m} / \mathrm{min}$ at $15^{\circ}$ ), showed only a modest degree of hypertrophy (Zhang et al. 2002). Thus, it is conceivable that the accumulated time at a high aerobic intensity accentuates cardiac hypertrophy and/or that anaerobic intensities may also cause counterproductive responses.

Although most of the studies suggest that the growth response of the LV is greater or equal to the RV, it has also been reported that RV hypertrophy may be greater (Anversa et al. 1983). This may be explained by the RV performing greater relative work during exercise because of smaller mass, thinner wall, and fewer cardiomyocytes, compared to the LV.

\section{Voluntary wheel running}

Voluntary running programs carried out on running wheels with either no or various degrees of resistance offer less control of the exercise, since running periods and effort levels are determined by the animal itself, and may only be recorded and limited, but not reliably instigated, by the researchers. Nonetheless, voluntary wheel running has been reported to induce robust physiological hypertrophy (Allen et al. 2001, Konhilas et al. 2004, Moraska et al. 2000, Natali et al. 2001), demonstrating sufficient inherent motivation to induce adaptation. However, voluntary daily running distance peaks after $\sim 2-4$ weeks at $\sim 10-15 \mathrm{~km} /$ day, and thereafter declines to $<4 \mathrm{~km} /$ day. Accordingly, complete hypertrophy has been observed after only 3-4 weeks of voluntary wheel running, whereas longer exercise training programs have not produced further hypertrophy (Natali et al. 2001, Yancey and Overton 1993).

\section{Swim training}

Swim training is initiated by placing animals in water tanks for a given period of time. The exercise load may be regulated by attaching weights or floating devices to the animals. Although the duration of the swim exercise has varied considerably; 1-6 hours/day and 1-24 months, it generally induces cardiac hypertrophy by as much as $15 \%$ (Kaplan et al. 1994, Medeiros et al. 2004), observable already after 1 hour/day of 1 week swim training (Edwards 2002). It has also been demonstrated that duration (60 vs. 90 minutes) per session or of the program (4 vs. 6 weeks) or frequency (1 vs. 2/week) may not affect the magnitude of the response, whereas external weights ( +2 vs. $+4 \%$ of body weight) in contrast may affect the response, with the heavier load being more effective due to greater cell hypertrophy (Evangelista et al. 2003). Hence, swim training appears equally effective as treadmill or voluntary wheel running programs for inducing physiological hypertrophy.

Water temperature, by regulating core temperature, has been observed to affect the physiological hypertrophy non-linearly. Swim training at $25{ }^{\circ} \mathrm{C}$ induced greater cardiac hypertrophy than at $35^{\circ} \mathrm{C}$ in young rats, but the opposite was true in old rats (Prathima and Devi 1999). However, varying the water temperature $30-36{ }^{\circ} \mathrm{C}$ yielded similar hypertrophy responses, whereas swim training at $38{ }^{\circ} \mathrm{C}$ failed to induce hypertrophy (Harri and Kuusela 1986). Water tank depth, density of animals, and water movement may also affect the outcome (Abel 1994, Iemitsu et al. 2003).

\section{Larger animal models of exercise training}

Larger mammalian models are frequently used to study cardiac structure and function. In general, these models resemble the human heart more accurately than rodents, in terms of oxygen uptake $\left(V \mathrm{O}_{2}\right)$ kinetics, cardiac mechanics, excitation-contraction coupling, force production, collateral coronary circulation, and subcellular architecture (Hasenfuss 1998, Hamilton and Ianuzzo 1991, White et al. 1987). Larger animals are also easier to instrument, but are costly, require substantial maintenance resources, have longer gestation periods, 
and do not currently allow for much genetic manipulation.

\section{Swine models of exercise training}

Treadmill running has been the preferred exercise mode in swine. Twelve weeks of various exercise training protocols, characterized by progressively increasing treadmill speeds and running durations, induced moderately increased heart and LV masses in Yucatan miniature and regular farm swine in an exercise intensity-dependent manner (Hinken et al. 2006, Laughlin et al. 1991). Longitudinal LV and cardiomyocyte cross-sectional areas and the LV cavity dimensions increased with exercise training, while the mitochondria and cell membranes adapted accordingly to maintain the cellular architecture. In another study, exercise training-induced increases in end-diastolic dimensions and stroke volumes were accounted for by reduced peripheral resistance and increased cardiac dimensions, and not by contractile performance (White et al. 1997). Intensity-controlled exercise has not been conducted in swine, but protocols for measuring $V \mathrm{O}_{2 \max }$ have been published (Jorgensen et al. 2009).

\section{Dog models of exercise training}

Although exercise training reduces resting heart rates and heart rate responses to exercise (Carew and Covell 1978, Stepien et al. 1998), the reported morphological changes include increased (Wyatt and Mitchell 1974) or unchanged (Ritzer et al. 1980) LV wall dimensions after exercise training in non-greyhound breeds of dogs. This lack of consistency is likely due to the fact that many of the dog exercise training results are stemming from uncontrolled studies. Few of them are experimentally controlled, and rather compare race- or work-dogs to sedentary counterparts. Species differences and environmental factors may also have confounded the results. Nevertheless, LV mass and dimensions, and cardiomyocyte diameter were up to $50 \%$ greater in racetrained greyhounds than in normal size-matched dogs (Carew and Covell 1978). Similarly, 5 months of $\sim 20 \mathrm{~km} /$ day loaded sled pulling in Alaskan sled dogs increased LV diameters and mass and reduced resting heart rates, although no changes in echocardiographyderived measures of LV function were found (Stepien et al. 1998). However, disproportionate LV wall thickening and dilatation suggested the changes were due to volume and pressure overloading; as expected from combined isometric and isotonic exercise training.

\section{Rabbit models of exercise training}

Rabbit models are commonly used for myocardial studies in both health and disease. They are less expensive than other large animals, have important similarities to the human heart, and show pathologic cardiac remodeling that closely resembles that of humans (Hasenfuss 1998). Several studies have reported exercise in New Zealand white rabbits, by using the low-speed flat treadmill $(18-20 \mathrm{~m} / \mathrm{min})$ for $40-60 \mathrm{~min} /$ day as the exercise stimulus (Carroll and Kyser 2002, Gao et al. 2007). Physiological hypertrophy has not been firmly established in rabbits, but indices of myocardial hypertrophy have been reported, by measuring endsystolic pressure-length relationships after catheteroccluding aortic blood. After exercise training, the slopes for the longitudinal segments of the LV were less steep than in sedentary control rabbits, although shortening of the segments were similar. This suggests that the myocardium may have structurally remodeled (Hexeberg et al. 1995).

\section{Advantages and disadvantages of different modes of exercise training}

The precise control of exercise intensity and volume that allows for uniform and well-controlled exercise workloads (duration, inclination, speed, distance) can only be achieved by the use of treadmills (Hoydal et al. 2007). Moreover, only treadmills offer a reliable mode by which exercise and cardiovascular parameters can be measured under controlled conditions. This also leads to a low variation within the experimental groups and thus enables us to detect small changes (Kemi et al. 2004). Hence, treadmill running has become a preferred option for controlled studies of the effects of exercise training, despite it being labor-intensive and requiring of technical skills and specialized equipment. However, treadmill running may present difficulty for motivating animals for prolonged exercise, and animals may experience physical and psychological stress from forced running. The stress responses have included reduced peripheral thyroid hormone levels and induction of adrenal hypertrophy, thymic involution, reduced serum corticosteroid binding globulin levels, and total adrenal medullary epinephrine contents (Helmreich et al. 2005, Moraska et al. 2000). On the other hand, treadmill running programs also induce numerous functional and structural beneficial adaptive responses in multiple organ systems (Haram et al. 2009, Kemi et al. 2007, 2008). 
It is unclear to what degree the stress reactions are attributed to forced or voluntary exercise training, since also voluntary exercise training programs activate the stress response (Droste et al. 2007). In this study, voluntary wheel trained mice also showed lower earlymorning plasma adrenocorticotropic hormone levels and substantially higher plasma corticosterone levels compared to controls. Heavier adrenal glands due to an enlarged adrenal cortex were also reported, whereas the adrenal medulla expressed higher levels of tyrosine hydroxylase mRNA, indicating higher sympathoadrenomedullary activity. Thus, it indicates that both hypothalamic-pituitary-adrenal and the sympathoadrenomedullary systems may be regulated by voluntary wheel running. Therefore, the possibility arises that stress reactions are normal or unavoidable in response to exercise training, and that this does not prevent physiological hypertrophy. For instance, no signs of perturbation to regular exercise and cardiovascular parameters after forced exercise training have been detected, and no pathological signaling events have been observed after forced swim or treadmill exercise training programs (McMullen et al. 2004). In fact, hypothalamic-pituitary-adrenal and sympathoadrenomedullary activation may also contribute to physiological cardiac hypertrophy (Kurowski et al. 1984).

A potential benefit of voluntary wheel running is that it allows for activity in a non-stressful environment on the animal's own premises with only minimal technical requirements. However, since the average spontaneous running distance and pattern may vary extensively between animals (Lambert et al. 1996), it consequently leads to heterogeneous effect magnitudes. Moreover, voluntary wheel running provides fewer details of activity levels and may only indirectly measure exercise performance.

A main advantage of swim training is that a relatively large number of animals can be exercised readily and simultaneously, and yet without depending on animal self-motivation, although efficient swimmers may lower their activity levels unless the exercise load is increased. However, the aqueous environment may add additional stress to the animal, such as diving-related hypoxia, Valsalva maneuvers, feeling of discomfort, and fear of drowning, and low water temperatures may lead to changes in the neuroendocrine system as well as hemodynamic changes due to increased blood pressure (Kioukia-Fougia et al. 2002). Furthermore, swim training is associated with the difficulty of quantifying exercise intensity because of the lack of graded workload protocols and water interfering with the recording equipment. Nonetheless, swim training induces chronic cardiac hypertrophy with a molecular signature indicating physiological activation of growth signaling (Kemi et al. 2008, McMullen et al. 2004).

Finally, it should be noted that the control for exercise training effects in animals is accomplished by studying similar animals that are forced to remain sedentary over the course of the study. However, this deviates from the human situation where most individuals engage in at least some form of physical exercise, although it may be at a very low level. Currently, it is impossible to quantify how this affects the outcome of research studies, but it seems likely that both exercise trained and sedentary animals constitute the two extreme ends of physical activity; close-tocomplete sedentary or highly active lifestyles, whereas most humans that are not physically constrained from physical exercise find themselves somewhere between those endpoints.

\section{Advantages and disadvantages of different species}

Important differences and therefore similarities and dissimilarities to human hearts have been referred to above; factors that effectively constitute important advantages and disadvantages associated with the choice of animal model. In short, the hearts of larger animals display more similarities to human hearts than small rodents, but require more resources and larger facilities in terms of purchase, breeding, maintenance, exercise training protocols, and final experiments, whether the latter is of whole-body function or through instrumentation or isolation of tissues or cells. Moreover, larger animals are more difficult to manipulate than small rodents, and at this stage, less is known about the exercise patterns or willingness to exercise in larger animals compared to rodents. For instance, the different mode of locomotion in rabbits, especially during stress, needs to be accommodated for when designing exercise training protocols. Nonetheless, the possibility remains that growth patterns are well conserved between species. However, substantial evidence suggests that differences in exercise patterns may not only occur between species, but also between different strains within the same species; see below for more details. 


\section{Factors influencing exercise training-induced cardiac hypertrophy}

Age

Aging is associated with a diminished capacity for myocardial hypertrophy, as exemplified by smaller magnitudes of change in old compared to young rats (Anitha and Devi 1996). This may partly be due to a greater cardiomyocyte proliferation in early life (Bergmann et al. 2009), which has not been shown in aged hearts. Exercise training also protects against the aging-induced decrease in arterial elasticity, and thereby reduces the development of cardiac pathology (Rosa et al. 2005).

Sex

Sex is a dominant factor for cardiac adaptation. For instance, female mice of both $\mathrm{C} 57 \mathrm{BL} / 6 \mathrm{~J}$ and $\mathrm{FVB} / \mathrm{NJ}$ strains exhibit a greater relative gain in cardiac mass than male mice after voluntary wheel running (Konhilas et al. 2004). When the gain in cardiac mass was normalized for cage wheel activity, C57BL/6J females had an augmented hypertrophic response for the given physiologic stimulus, compared to males. This is also true for treadmill running programs in both mice and rats, albeit not at the cell level (Kemi et al. 2002, Wisloff et al. 2001). The sexdependence of adaptation was larger than the straindependence, indicating sexually dimorphic hypertrophy mechanisms. Running distances peaking every 4 days, regardless of the weekly average, suggests that the estrous cycle may affect either running performance or hypertrophy response in female animals (Natali et al. 2001).

\section{Strain}

Rats of similar genetic stock, even littermates, may have as much as a 50 -fold difference in daily running distances (Lambert et al. 1996). A lack of correlation between treadmill running and voluntary wheel running performances (Lerman et al. 2002) indicates that exercise performance is a complex, multifactorial trait. Cardiac differences between both rat and mouse strains may contribute to the differences in aerobic capacity (Allen et al. 2001, Barbato et al. 1998, Koch et al. 1999, Lerman et al. 2002). For instance, Dark Agouti rats have both greater treadmill running capacity and heart:body weight ratios than Copenhagen rats, and similar strain differences exist in mice. Hence, within both mice and rats, some strains have inherently different exercise capacities, which may also contribute to distinct cardiac adaptations to exercise training.

\section{Exercise intensity}

The importance of exercise intensity is evidenced by studies showing that high aerobic intensity of the exercise training yields a larger magnitude of effects than low-to-moderate exercise intensity, including the cardiac hypertrophy (Haram et al. 2009, Kemi et al. 2002). No threshold intensity of exercise for inducing observable cardiac hypertrophy has yet been identified, but cardiac hypertrophy is only sustained if exercise is sustained, and lost within weeks if exercise training is not continued (Kemi et al. 2004).

The gold standard for monitoring exercise intensity is by live recording of $V \mathrm{O}_{2}$ and $V \mathrm{O}_{2 \max }$. Albeit laborious, appropriate protocols have been established in several species (Jorgensen et al. 2009, Kemi et al. 2002, Wisloff et al. 2001). Other measures of aerobic fitness, such as heart rate, blood pressure, lactate concentration and running speed do not offer the same validity, and often require immobilization and surgical procedures that may confound the measurements.

\section{Cell signaling of cardiac hypertrophy}

Although this review does not focus on the signaling pathways that associate with exercise-induced cardiac hypertrophy, it should be noted that several exercise modes have strongly indicated that the insulinlike growth factor-1 (IGF-1)-induced Akt-mammalian target of rapamycin (mTOR) pathway at least partly controls physiological hypertrophy, and in fact, activation or deactivation of this pathway may differentiate physiological from pathological hypertrophy (Kemi et al. 2008, McMullen et al. 2004). However, it is clear that genome-wide genetic regulation (Beisvag et al. 2009) as well as activation levels of specific pathways controlled by for instance calcineurin, endothelin, and fetal genes also contribute toward such differentiation (Hunter and Chien 1999, Wilkins et al. 2004). Thus, various animal models and modes of exercise training have been used to consistently identify molecular pathways that control cardiac hypertrophy and that contrast physiological from pathological hypertrophies. Exactly why physiological hypertrophy is molecularly distinct from pathological hypertrophy is unknown, but the different nature of the stress to the heart imposed by exercise 
compared to for instance that imposed by acute injury, chronic hypertension, or chronic dilatation presumably controls this.

\section{Measurement of cardiac hypertrophy}

\section{Cardiac weights}

The most common index of cardiac hypertrophy is the measure of heart or ventricular weights related to body weight. However, relating heart to body weights is based upon several assumptions. If several groups are compared, it is only valid if the groups exhibit similar body growth patterns. For instance, body weight and composition may also change with exercise training and may vary between sexes, as male rats reduced body mass more than females after exercise training, due to a greater loss of body fat, regardless of the mode or duration of exercise (Pitts 1984). Thus, increased heart:body weight ratio may also reflect a conserved heart weight if fat mass is reduced or body growth is suppressed during exercise training. Therefore, heart and ventricular weights should be related to lean body mass or to an allometrically scaled body mass (Batterham et al. 1997, Whalley et al. 1999).

Because of fluctuating body weights, cardiac hypertrophy has also been normalized to tibial length; considered a static parameter in mature animals. Histology in maturing rats agreed more with heart weight:tibia length than the heart:body weight ratio, indicating that body weight may be inaccurate for quantifying cardiac hypertrophy unless it is properly scaled (Yin et al. 1982).

\section{Ultrasound echocardiography}

Although non-invasive ultrasound echocardiography has enabled characterization of pathological hearts (Wisloff et al. 2002), it has only been of limited value with exercise training. Echocardiography assessments of exercise-induced cardiac hypertrophy have been less sensitive than post-mortem measurements. In both mice and rats, scanning with a $10 \mathrm{MHz}$ probe only partially detected cardiac hypertrophy after a treadmill running program that increased heart:body weight ratios and cardiomyocyte dimensions by $15-35 \%$ (Kemi et al. 2002, 2005). One of the limitations associated with echocardiography has been poor axial resolution, which in practice has been $\sim 2.2 \mathrm{~mm}$. This is close to what one may expect from a difference between exercise trained and sedentary LV end-diastolic diameter and LV posterior wall thickness in rodents; main parameters for evaluating cardiac hypertrophy by echocardiography. However, this may change with the new generation of high-resolution echocardiography.

\section{Single isolated cardiomyocytes}

Exercise training-induced cardiac hypertrophy is mainly brought about by increased cardiomyocyte dimensions. Accordingly, cardiomyocyte hypertrophy has been reported after voluntary wheel running, swim training, and treadmill running programs in both rats and mice (Kemi et al. 2002, McMullen et al. 2004, Moran et al. 2003, Wisloff et al. 2002). Some reports suggest that the changes in the cardiomyocyte dimensions are specific to the region from which they are isolated (Natali et al. 2001), but cardiomyocytes isolated from non-specific LV areas have nonetheless provided enough sensitivity to establish differences in cellular morphology between various exercise training protocols (Kemi et al. 2005).

The experimental conditions under which cardiomyocytes are dissociated and measured, especially ionic concentrations, should be accurately controlled, since diastolic contraction, and hence read-out resting cell dimensions or volume-dependent capacitance, would depend on this.

\section{Conclusions}

Several modes of exercise training have been introduced to a number of animal models of various genetic backgrounds, such as treadmill running, voluntary wheel running, and swim training. These models lead to physiological hypertrophy, measured both at the whole heart and ventricle and individual cardiomyocyte level. Observable and significant hypertrophy can be detected already after $\sim 1$ month of exercise training, and reaches a plateau after a few months if the exercise training is sustained. It has not yet been established whether further hypertrophy can be induced after the plateau, by increasing the amount of exercise training by either intensity or volume. However, each of these models is associated with both advantages and disadvantages that should be considered when interpreting the research results and when choosing model systems for future studies. Although the use of rodents in exercise studies by far outnumbers the use of larger animals, specific research questions may necessitate experiments in larger animals, especially if similarity to human biology is important. Nonetheless, in our experience, exercise training in rodents also mimics the responses to exercise 
training observed in humans, as far as different species may be compared.

\section{Conflict of Interest}

There is no conflict of interest.

\section{References}

ABEL EL: Behavioral and physiological effects of different water depths in the forced swim test. Physiol Behav 56: 411-414, 1994.

ALLEN DL, HARRISON BC, MAASS A, BELL ML, BYRNES WC, LEINWAND LA: Cardiac and skeletal muscle adaptations to voluntary wheel running in the mouse. J Appl Physiol 90: 1900-1908, 2001.

ANITHA V, DEVI SA: Age-related responses of right ventricle in swim-trained rats: changes in lactate and pyruvate contents and lactate dehydrogenase activity. Mech Ageing Dev 90: 91-102, 1996.

ANVERSA P, LEVICKY V, BEGHI C, MCDONALD SL, KIKKAWA Y: Morphometry of exercise-induced right ventricular hypertrophy in the rat. Circ Res 52: 57-64, 1983.

BARBATO JC, KOCH LG, DARVISH A, CICILA GT, METTING PJ, BRITTON SL: Spectrum of aerobic endurance running performance in eleven inbred strains of rats. J Appl Physiol 85: 530-536, 1998.

BATTERHAM AM, GEORGE KP, MULLINEAUX DR: Allometric scaling of left ventricular mass by body dimensions in males and females. Med Sci Sports Exerc 29: 181-186, 1997.

BEISVAG V, KEMI OJ, ARBO I, LOENNECHEN JP, WISLOFF U, LANGAAS M, SANDVIK AK, ELLINGSEN O: Pathological and physiological hypertrophies are regulated by distinct gene programs. Eur J Cardiovasc Prev Rehabil 16: 690-697, 2009.

BELLAFIORE M, SIVVERINI G, PALUMBO D, MACALUSO F, BIANCO A, PALMA A, FARINA F: Increased cx43 and angiogenesis in exercised mouse hearts. Int J Sports Med 28: 749-755, 2007.

BERGMANN O, BHARDWAJ RD, BERNARD S, ZDUNEK S, BARNABE-HEIDER F, WALSH S, ZUPICICH J, BUCHHOLZ BA, DRUID H, JOVINGE S, FRISEN J: Evidence for cardiomyocyte renewal in humans. Science 324: 98-102, 2009.

CAREW TE, COVELL JW: Left ventricular function in exercise-induced hypertrophy in dogs. Am J Cardiol 42: 82-88, 1978.

CARROLL JF, KYSER CK: Exercise training in obesity lowers blood pressure independent of weight change. $M e d ~ S c i$ Sports Exerc 34: 596-601, 2002.

DIFFEE GM, NAGLE DF: Regional differences in effects of exercise training on contractile and biochemical properties of rat cardiac myocytes. J Appl Physiol 95: 35-42, 2003.

DROSTE SK, CHANDRAMOHAN Y, HILL LE, LINTHORST AC, REUL JM: Voluntary exercise impacts on the rat hypothalamic-pituitary-adrenocortical axis mainly at the adrenal level. Neuroendocrinology 86: 26-37, 2007.

EDWARDS JG: Swimming training increases ventricular atrial natriuretic factor (ANF) gene expression as an early adaptation to chronic exercise. Life Sci 70: 2753-2768, 2002.

EVANGELISTA FS, BRUM PC, KRIEGER JE: Duration-controlled swimming exercise training induces cardiac hypertrophy in mice. Braz J Med Biol Res 36: 1751-1759, 2003.

FENNING A, HARRISON G, DWYER D, ROSE'MEYER R, BROWN L: Cardiac adaptation to endurance exercise in rats. Mol Cell Biochem 251: 51-59, 2003.

FEWELL JG, OSINSKA H, KLEVITSKY R, NG W, SFYRIS G, BAHREHMAND F, ROBBINS J: A treadmill exercise regimen for identifying cardiovascular phenotypes in transgenic mice. Am J Physiol 273: H1595H1605, 1997.

GAO L, WANG W, LIU D, ZUCKER IH: Exercise training normalizes sympathetic outflow by central antioxidant mechanisms in rabbits with pacing-induced chronic heart failure. Circulation 115: 3095-3102, 2007.

HAMILTON N, IANUZZO CD: Contractile and calcium regulating capacities of myocardia of different sized mammals scale with resting heart rate. Mol Cell Biochem 106: 133-141, 1991.

HARAM PM, KEMI OJ, LEE SJ, BENDHEIM MO, AL-SHARE QY, WALDUM HL, GILLIGAN LJ, KOCH LG, BRITTON SL, NAJJAR SM, WISLOFF U: Aerobic interval training vs. continuous moderate exercise in the metabolic syndrome of rats artificially selected for low aerobic capacity. Cardiovasc Res 81: 723-732, 2009. 
HARRI M, KUUSELA P: Is swimming exercise or cold exposure for rats? Acta Physiol Scand 126: 189-197, 1986.

HASENFUSS G: Animal models of human cardiovascular disease, heart failure and hypertrophy. Cardiovasc Res 39: 60-76, 1998.

HELMREICH DL, PARFITT DB, LU XY, AKIL H, WATSON SJ: Relation between the hypothalamic-pituitarythyroid (HPT) axis and the hypothalamic-pituitary-adrenal (HPA) axis during repeated stress. Neuroendocrinology 81: 183-192, 2005.

HEXEBERG E, WESTBY J, HESSEVIK I, HEXEBERG S: Effects of endurance training on left ventricular performance: a study in anaesthetized rabbits. Acta Physiol Scand 154: 479-488, 1995.

HINKEN AC, KORTE FS, MCDONALD KS: Porcine cardiac myocyte power output is increased after chronic exercise training. J Appl Physiol 101: 40-46, 2006.

HOYDAL MA, WISLOFF U, KEMI OJ, ELLINGSEN O: Running speed and maximal oxygen uptake in rats and mice: practical implications for exercise training. Eur J Cardiovasc Prevent Rehabil 14: 753-760, 2007.

HUNTER JJ, CHIEN KR: Signaling pathways for cardiac hypertrophy and failure. New Engl J Med 341: 1276-1283, 1999.

IEMITSU M, MAEDA S, OTSUKI T, GOTO K, MIYAUCHI T: Time course alterations of myocardial endothelin-1 production during the formation of exercise training-induced cardiac hypertrophy. Exp Biol Med 231: 871-875, 2006.

IEMITSU M, MIYAUCHI T, MAEDA S, SAKAI S, FUJII N, MIYAZAKI H, KAKINUMA Y, MATSUDA M, YAMAGUCHI I: Cardiac hypertrophy by hypertension and exercise training exhibits different gene expression of enzymes in energy metabolism. Hypertens Res 26: 829-837, 2003.

JORGENSEN A, BERGE VJ, BRUBAKK AO, WISLOFF U: A reliable and valid protocol for measuring maximal oxygen uptake in pigs. Eur J Cardiovasc Prev Rehabil 16: 628-632, 2009.

KAPLAN ML, CHESLOW Y, VIKSTROM K, MALHOTRA A, GEENEN DL, NAKOUZI A, LEINWAND LA, BUTTRICK PM: Cardiac adaptations to chronic exercise in mice. Am J Physiol 267: H1167-H1173, 1994.

KEMI OJ, LOENNECHEN JP, WISLOFF U, ELLINGSEN O: Intensity-controlled treadmill running in mice: cardiac and skeletal muscle hypertrophy. J Appl Physiol 93: 1301-1309, 2002.

KEMI OJ, HARAM PM, WISLOFF U, ELLINGSEN O: Aerobic fitness is associated with cardiomyocyte contractile capacity and endothelial function in exercise training and detraining. Circulation 109: 2897-2904, 2004.

KEMI OJ, HARAM PM, LOENNECHEN JP, OSNES JB, SKOMEDAL T, WISLOFF U, ELLINGSEN O: Moderate vs. high exercise intensity: differential effects on aerobic fitness, cardiomyocyte contractility, and endothelial function. Cardiovasc Res 67: 161-172, 2005.

KEMI OJ, ELLINGSEN O, CECI M, GRIMALDI S, SMITH GL, CONDORELLI G, WISLOFF U: Aerobic interval training enhances cardiomyocyte contractility and $\mathrm{Ca}^{2+}$ cycling by phosphorylation of CaMKII and Thr-17 of phospholamban. J Mol Cell Cardiol 43: 354-361, 2007.

KEMI OJ, CECI M, CONDORELLI G, SMITH GL, WISLOFF U: Myocardial sarcoplasmic reticulum $\mathrm{Ca}^{2+} \mathrm{ATPase}$ function is increased by aerobic interval training. Eur J Cardiovasc Prev Rehabil 15: 145-148, 2008 a.

KEMI OJ, CECI M, WISLOFF U, GRIMALDI S, GALLO P, SMITH GL, CONDORELLI G, ELLINGSEN O: Activation or inactivation of cardiac Akt/mTOR signaling diverges physiological from pathological hypertrophy. J Cell Physiol 214: 316-321, 2008 b.

KIOUKIA-FOUGIA N, ANTONIOU K, BEKRIS S, LIAPI C, CHRISTOFIDIS I, PAPADOPOULOU-DAIFOTI Z: The effects of stress exposure on the hypothalamic-pituitary-adrenal axis, thymus, thyroid hormones and glucose levels. Prog Neuropsychopharmacol Biol Psychiatry 26: 823-830, 2002.

KOCH LG, BRITTON SL, BARBATO JC, RODENBAUGH DW, DiCARLO SE: Phenotypic differences in cardiovascular regulation in inbred rat models of aerobic capacity. Physiol Genomics 1: 63-69, 1999.

KONHILAS JP, MAASS AH, LUCKEY SW, STAUFFER BL, OLSON EN, LEINWAND LA: Sex modifies exercise and cardiac adaptation in mice. Am J Physiol 287: H2768-H2776, 2004.

KUROWSKI TT, CHATTERTON RT Jr, HICKSON RC: Glucocorticoid-induced cardiac hypertrophy: additive effects of exercise. $J$ Appl Physiol 57: 514-519, 1984.

LAMBERT MI, VAN ZYL C, JAUNKY R, LAMBERT EV, NOAKES TD: Tests of running performance do not predict subsequent spontaneous running in rats. Physiol Behav 60: 171-176, 1996. 
LAUGHLIN MH, HALE CC, NOVELA L, GUTE D, HAMILTON N, IANUZZO CD: Biochemical characterization of exercise-trained porcine myocardium. J Appl Physiol 71: 229-235, 1991.

LERMAN I, HARRISON BC, FREEMAN K, HEWETT TE, ALLEN DL, ROBBINS J, LEINWAND LA: Genetic variability in forced and voluntary endurance exercise performance in seven inbred mouse strains. $J \mathrm{Appl}$ Physiol 92: 2245-2255, 2002.

MARON BJ, PELLICCIA A: The heart of trained athletes: cardiac remodeling and the risks of sports, including sudden death. Circulation 114: 1633-1644, 2006.

MCMULLEN JR, SHIOI T, ZHANG L, TARNAVSKI O, SHERWOOD MC, DORFMAN AL, LONGNUS S, PENDE M, MARTIN KA, THOMAS G, IZUMO S: Deletion of ribosomal S6 kinases does not attenuate pathological, physiological, or insulin-like growth factor 1 receptor-phosphoinositide 3-kinase-induced cardiac hypertrophy. Mol Cell Biol 24: 6231-6240, 2004.

MEDEIROS A, OLIVEIRA EM, GIANOLLA R, CASARINI DE, NEGRAO CE, BRUM PC: Swimming training increases cardiac vagal activity and induces cardiac hypertrophy in rats. Braz J Med Biol Res 37: 1909-1917, 2004.

MOORE RL, MUSCH TI, YELAMARTY RV, SCADUTO RC Jr, SEMANCHICK AM, ELENSKY M, CHEUNG JY: Chronic exercise alters contractility and morphology of isolated rat cardiac myocytes. Am J Physiol 264: C1180-C1189, 1993.

MORAN M, SABORIDO A, MEGIAS A: $\mathrm{Ca}^{2+}$ regulatory systems in rat myocardium are altered by 24 weeks treadmill training. Pflugers Arch 446: 161-168, 2003.

MORASKA A, DEAK T, SPENCER RL, ROTH D, FLESHNER M: Treadmill running produces both positive and negative physiological adaptations in Sprague-Dawley rats. Am J Physiol 279: R1321-R1329, 2000.

NATALI AJ, TURNER DL, HARRISON SM, WHITE E: Regional effects of voluntary exercise on cell size and contraction-frequency responses in rat cardiac myocytes. $J$ Exp Biol 204: 1191-1199, 2001.

PITTS GC: Body composition in the rat: interactions of exercise, age, sex, and diet. Am J Physiol 246: R495-R501, 1984.

PRATHIMA S, DEVI SA: Adaptations in lactate dehydrogenase and its isozymes in aging mammalian myocardium: interaction of exercise and temperature. Mech Ageing Dev 108: 61-75, 1999.

RITZER TF, BOVE AA, CAREY RA: Left ventricular performance characteristics in trained and sedentary dogs. J Appl Physiol 48: 130-138, 1980.

ROSA EF, SILVA AC, IHARA SS, MORA OA, ABOULAFIA J, NOUAILHETAS VL: Habitual exercise program protects murine intestinal, skeletal, and cardiac muscles against aging. $J$ Appl Physiol 99: 1569-1575, 2005.

STEPIEN RL, HINCHCLIFF KW, CONSTABLE PD, OLSON J: Effect of endurance training on cardiac morphology in Alaskan sled dogs. J Appl Physiol 85: 1368-1375, 1998.

WHALlEY GA, GAMBLE GD, DOUGHTY RN, CULPAN A, PLANK L, MACMAHON S, SHARPE N: Left ventricular mass correlates with fat-free mass but not fat mass in adults. J Hypertens 17: 569-574, 1999.

WHITE FC, MCKIRNAN MD, BREISCH EA, GUTH BD, LIU YM, BLOOR CM: Adaptation of the left ventricle to exercise-induced hypertrophy. J Appl Physiol 62: 1097-1110, 1987.

WILKINS BJ, DAI YS, BUENO OF, PARSONS SA, XU J, PLANK DM, JONES F, KIMBALL TR, MOLKENTIN JD: Calcineurin/NFAT coupling participates in pathological, but not physiological, cardiac hypertrophy. Circ Res 94: 110-118, 2004.

WISLOFF U, HELGERUD J, KEMI OJ, ELLINGSEN O: Intensity-controlled treadmill running in rats: $\mathrm{VO}_{2 \max }$ and cardiac hypertrophy. Am J Physiol 280: H1301-H1310, 2001.

WISLOFF U, LOENNECHEN JP, CURRIE S, SMITH GL, ELLINGSEN O: Aerobic exercise reduces cardiomyocyte hypertrophy and increases contractility, $\mathrm{Ca}^{2+}$ sensitivity and SERCA-2 in rat after myocardial infarction. Cardiovasc Res 54: 162-174, 2002.

WISLOFF U, NAJJAR SM, ELLINGSEN O, HARAM PM, SWOAP S, AL-SHARE Q, FERNSTROM M, REZAEI K, LEE SJ, KOCH LG, BRITTON SL: Cardiovascular risk factors emerge after artificial selection for low aerobic capacity. Science 307: 418-420, 2005.

WYATT HL, MITCHELL JH: Influences of physical training on the heart of dogs. Circ Res 35: 883-889, 1974. 
YANCEY SL, OVERTON JM: Cardiovascular responses to voluntary and treadmill exercise in rats. J Appl Physiol 75: 1334-1340, 1993.

YIN FC, SPURGEON HA, RAKUSAN K, WEISFELDT ML, LAKATTA EG: Use of tibial length to quantify cardiac hypertrophy: application in the aging rat. Am J Physiol 243: H941-H947, 1982.

ZHANG XQ, SONG J, CARL LL, SHI W, QUERSHI A, TIAN Q, CHEUNG JY: Effects of sprint training on contractility and $\mathrm{Ca}^{2+}{ }_{\text {i }}$ transients in adult rat myocytes. $J$ Appl Physiol 93: 1310-1317, 2002. 